\title{
Ontologies in Amine Platform: Structures and Processes
}

\author{
Adil Kabbaj ${ }^{1}$, Karim Bouzouba ${ }^{2}$, Khalid El Hachimi ${ }^{1}$, Nabil Ourdani ${ }^{1}$ \\ ${ }^{1}$ INSEA, B.P. 6217, Rabat, Morocco \\ akabbaj@insea.ac.ma \\ ${ }^{2}$ EMI, Mohamed V University, Avenue Ibnsina B.P. 765 Rabat Morocco \\ karim.bouzoubaa@emi.ac.ma \\ http://sourceforge.net/projects/amine-platform
}

\begin{abstract}
The theoretical part of this paper presents and discusses the conflict between Ontology and Commonsense Knowledge (CK) in the context of Computer-Human Interaction/Communication. A resolution of this conflict is proposed, where Ontology is considered as a special case of CK (i.e. a formal CK). By this "rehabilitation" of Ontology to "CK Base", we extend "by the way" the meaning and scope of Ontology to include "CK-based Ontology". This new kind of ontology considers both definitional and schematic/situational knowledge. Moreover, we propose for this new kind of ontology an organizational scheme based on the use of Conceptual Structures (like Definition, Canon and Schematic Clusters). The technical part presents the "implementation" of this theoretical framework in Amine platform [14] and especially in its Ontology layer. Amine's ontology meta-model is presented as well as various Ontology/CK-Base related processes.
\end{abstract}

\section{Introduction}

In Metaphysic and Philosophy, Ontology is not concerned by our "Commonsense Knowledge about the World", but by "the quest of what is necessary true about the Physical World" [20-22, 4]. Knowledge should be discarded in favour of a truthful and formal description of the "essence" of the World.

In the last decade, Artificial Intelligence (AI) and Information Sciences scientists started with "an analogical use" of the term Ontology; viewed as a Terminological/Definitional Knowledge Base that guarantees an efficient sharing of (Large) Knowledge Bases (KB). Ontology is therefore viewed as a "terminological" support to (large) KB [10-12, 21, 7, 3, and 24].

This "analogical use" of the term/concept Ontology has been criticized by philosophers [20-22, 10-12, 3, 7, 24] who propose instead the use of "Formal Ontology" and a methodology suited for ontology design [21, 10-12].

"Applied Ontology", based on Formal Ontology, is now emerging as a new multidisciplinary field, with Semantic Web as the application domain "par excellence” [9, 3].

In general, Formal Ontology is proposed as a new approach to Computer-Human Interaction/Communication (CHI/C). However, "Full/Natural” (i.e. uncontrolled, 
unrestricted, unlimited) $\mathrm{CHI} / \mathrm{C}$ is also a central topic in Cognitive Science in general and in $\mathrm{AI}$ in particular. It is well established, from these sciences, that Commonsense Knowledge (CK) is a (if not the) vital aspect, in this "big/hard" problem.

There is therefore a conflict between two opposite approaches to the same domain (CHI/C): Formal Ontology, which is (in principle) knowledge-free, and Commonsense Knowledge based approach.

In the theoretical part of this paper (sections 2-3), the first author presents this problematical conflict and proposes the following solution: a/an (formal) ontology is considered as a special case of Commonsense Knowledge -CK-; formal ontology is a "formal CK": a rigorous, systematic, definitional and truthful CK.

By this "rehabilitation" of Ontology to "CK based perspective", we extend "by the way" the meaning and scope of Ontology to include "CK-based Ontology". This new kind of ontology considers both definitional and schematic/situational knowledge. Moreover, we propose for this new kind of ontology an organizational scheme based on the use of Conceptual Structures -CS- (like Definition, Canon and Schematic Clusters). More specifically, CSs are used to organize the meaning of (i.e. the CK related to) concept types and relations inside an Ontology.

The technical part of this paper (sections 4-5) presents the "implementation" of this theoretical framework in Amine platform [14] and especially in its Ontology layer. Amine's ontology meta-model is presented as well as various Ontology/CK-Base related processes.

\section{Ontology vs Commonsense Knowledge (CK)}

\section{Ontology, Formal Ontology and “Applied Ontology”}

As an inquiry in Metaphysic and Philosophy, Ontology is not concerned by our "CK about the World", but by "the quest of what is necessary true about the Physical World". It is concerned by "What are the types of objects that exist in the World?, What are the types of relations between these objects?” etc. [20-22, 10-12]. A type corresponds to the form/essence of a set/category of objects. These objects, with their types and relations, "exist" even if there is no human to observe them (Trees, Animals, Bus ... exist even if no human observes them) [20-22]. In this sense, these objects (and their forms/essences) are independent of human mind and they are different from concepts as considered in Cognitive Psychology, Artificial Intelligence (AI) and Cognitive Science [20-22]. Also, CK should not be considered in THIS context since the purpose of developing World Ontology is to specify the essence of the objects of the World, not to specify our CK about the World [20-22]. For instance, in THIS context, Ontology should contain the definition of the Type Bus but not the following schema: "typically, a Bus contains a set of about 50 passengers, it is the instrument of travel by those passengers at a speed less than or equal to 55 miles per hour, and it is the object of driving by some driver" [25 p. 129]. This schema represents a "generic/typical situation"; a CK about the type Bus. Several other schemata can be related to the type Bus (the same holds for any other type). A schema 
is not (necessarily) a domain-specific knowledge and not (necessarily) a linguistic construct (i.e. a "context of use" of a word); it may represent a generic situation or $\mathrm{CK}$, as illustrated by the above schema.

However, for the Metaphysician/Philosopher, this CK about the type Bus, even if it is used by human in his everyday life/activity and in his interaction/communication, is not relevant for his Ontology and should not be included in it, because it is not about the essence (the essential form) of "what is the type" Bus.

Conclusion: if the purpose is the construction/development of a World Ontology that should contain only the "essence" of the physical objects, then yes, CK should be discarded and ignored.

To complete this brief introduction to "Metaphysical/Philosophical perspective of Ontology", let us consider the following points:

1 In Metaphysic/Philosophy, World Ontology is considered along two perspectives [4, 20, 21]: (i) "Realist" perspective (revisionary metaphysics) which considers only the Physical World; it is concerned only by types of the physical objects, not by abstract types. Informally, we can say that "realist" perspective is interested by the "World without Human" (or Human as a simple physical object, among the other physical objects)! (ii) "Phenomenological" perspective (descriptive metaphysics) which considers the "Commonsense World" including physical objects created (and actions performed) by human and abstract types ("Commonsense World" should not be confused with the "CK about the Commonsense World").

2 Metaphysicians/Philosophers have developed various "Universal Ontologies" for the World in totality. They have developed also "Domain-specific Ontologies" like "Ontology for Physics", "Ontology for Biology", "Ontology for Medicine”, "Ontology for Math", etc. as well as "Ultra-domain-specific Ontologies" like "Ontology for Emotion”, “Ontology for Human Action”, etc.

3 Metaphysicians/Philosophers/Logicians have used/developed formal tools (formal logic, formal semantic, set theory, mereology, topology, etc.) for the formulation of their ontologies. They have developed "Formal Ontology" [20, 21, 10, and 11]. The philosopher Cocchiarella defines "Formal Ontology" as "the systematic, formal, axiomatic development of the logic of all forms and modes of being" [11]. Guarino insists that "formal" in this definition means "rigorous" and "related to the forms of being". Guarino concludes that Formal Ontology is concerned by the rigorous description of the forms (i.e. essence) of the physical Objects. He adds that in practice, Formal Ontology can be intended as the theory of a priori distinctions: (i) among the entities of the world (physical objects, events, regions, quantities of matter...); (ii) among the meta-level categories used to model the world (concepts, properties, qualities, states, roles, parts...).

Guarino proposed "ontological principles" that "operationalize" the above "a priori distinctions" [10, 11]. He developed also a methodology for "Ontology design" based on these principles. He proposed these principles as a foundation for the "Ontology Level" of any Knowledge Representation system [12].

1 Metaphysicians/Philosophers/Logicians have used computer science (and some AI tools and techniques) for the implementation of their ontologies. The Basic 
Formal Ontology (BFO) framework, in which the philosopher B. Smith (and other philosophers) is involved, is an example of this "Applied Ontology".

2 With this new interest to Ontology, it became clear that any domain could/should have its ontology formally defined and implemented (using hopefully the methodology proposed by Guarino). Computer scientists, involved in Information Sciences (Data Bases, Information Systems, Semantic Web [3, 9], etc.), as well as AI scientists, involved in the development of Large/Sharable Knowledge Bases, have developed several ontologies, producing a new field that can be called "Applied Ontology" (this is the name of a new Journal edited by Guarino).

In conclusion, Formal Ontology should be used to develop ontologies for ComputerComputer Interaction/Communication (CCI/C) or when human is involved in a very limited, controlled and restricted way.

\section{Commonsense Knowledge (CK) in Cognitive Psychology/Science}

Aristotle defined Human as "Animal with Rationality". Maybe a better definition would be "Animal with Knowledge". Human intelligence and cognition is based on his Conceptual System, Knowledge, Memory and, of course, the related cognitive processes. This is the main conclusion from Cognitive Science and AI ([6, 8, 15-19, 23-25] ... it is nonsensical to cite in this paper the huge literature that confirms this conclusion). Natural language, Human behaviour, thinking and reasoning, Human interaction and communication ... can't be treated adequately without taking into account (and seriously) this centrality of Knowledge.

Following Kant, Peirce, Whitehead and the mainstream of AI and Cognitive Science, we may say that "Human IS Process/Knowledge", the World for Human IS his "World of Processes/Knowledge" which includes his CK about the "commonsense World". And his "direct perception" of this "commonsense World" may be a "faithful image" of the Physical World [20-22], but an image that is included (immersed) in his CK.

At a more concrete level, "Knowledge about the commonsense World" can be viewed as a subset (a fragment) of Human Knowledge (even if this view is very simplistic; Knowledge about Commonsense World can't be clearly separated from the other Knowledge "parts").

CK describes generic (and typical) knowledge about the "commonsense World". CK is composed of definitional Knowledge AND situational/typical knowledge. A CK Base may contain for instance, the definition of the concept Bus AND a "schematic cluster" for concept Bus. Schematic cluster is a collection of schemata like the schema for Bus presented before.

A schema represents (in general) a generic situation. It provides (in general) default knowledge and it is an important source of expectation and prediction. It may provide causal knowledge, explanation knowledge, axiomatic/constraint and rule based knowledge, procedural knowledge, strategic knowledge, emotional knowledge, etc.

Often, human is unable to provide a precise definition of a type. An ontologist will consider such a type as a primitive type and no related knowledge (especially CK) is specified in the Ontology. From the knowledge-perspective view, this position is too 
restrictive. CK, in terms of schematic clusters related to concept types, are a valuable source of knowledge, for primitive types as well as for defined types.

Schema Theory is a central component in Cognitive Science and AI [6, 8, 15-19, 2325]. It is however outside the scope of this paper.

The important point here is that "What is the World?" for "ordinary" Human (not for the Metaphysician/Philosopher) IS the CK. In other words: what is refereed by Metaphysician/Philosopher as "Ontology" is refereed by "ordinary" Human as CK. Human, in his everyday life and activity, is not concerned by the "essence" of the objects or by "what is necessary true about the World or about a specific domain". He is concerned by his knowledge about the World (or knowledge about a specific domain). In his use/practice of natural language, in his activity, in his reasoning, in his problem solving activity, in his interaction and communication, etc., "ordinary" human uses his CK, not a Metaphysical/Philosophical ontology.

In Human-Human Interaction/Communication, the situation is very clear: the shared knowledge is not a "metaphysical/formal ontology" but CK.

The situation becomes problematic however, when the application domain concerns Computer-Human Interaction/Communication (CHI/C): Which approach to consider: "Formal Ontology" or CK?

\section{CHI/C: between Formal Ontology and Commonsense Knowledge}

Human, in his communication and interaction with human or with computer, uses his CK. Therefore, the above question becomes: In the context of "real/natural" CHI/C, does the Computer (the intelligent system or agent) needs a "Formal Ontology Base" or a "CK Base"? Computer and Human should use/share similar Knowledge. It is therefore clear that the Computer should have also a CK Base (not a "Formal Ontology").

CK Base has a long history in AI. The importance of CK for (all) intelligent activities is strongly established. Different approaches have been (and are being) developed in AI to deal with (build and develop) CK. Among these approaches: (a) acquisition from natural language, (b) learning, (c) building a huge CK Base.

$\mathrm{Cyc}^{1}$ is the best example of building a huge CK Base. It is build however with an "ad hoc" approach [11]. In Cyc, CK is represented by a partitioned hierarchy and a flat collection of axioms. Our proposition is to organize CK in terms of Conceptual Structures (especially Definition and Schematic Cluster) around concept types and relations. This is crucial for a more modular AND "cognitively-inspired" organization.

Another problematic treatment of CK is encountered in the very used KL-ONE-like family (and Description Logic) [2, 1]. For instance, our schema for Bus should not be inserted in TBox since this Knowledge Base concerns only intensional/definitional knowledge. And it should not be inserted in ABox since this Knowledge Base concerns only assertional/extensional knowledge; knowledge which is specific to a particular problem [1, p. 12] (or a particular "state of affairs"). As noted before, our

${ }^{1}$ http://www.cyc.com/cyc 
schema for Bus (and many others) is not specific to a particular problem; it is a general knowledge. In fact, Brachman uses this same expression ("general knowledge") to refer to TBox [2]. However, the most part of "general knowledge" belongs to CK, which has no (clear) places in TBox/ABox scheme. Related critics to KL-ONE-like family (and to Description Logic) have been formulated by Doyle and Patil [5].

Let us recapitulate: we started this section with the question "Which approach to consider for 'real/natural' CHI/C: 'Formal Ontology' or CK?” The answer is "the use of CK”. But is it sufficient to share the same (or similar) content of CK (between Computer and Human) in order to guarantee a "natural-like" Interaction/ Communication? For instance, is it sufficient to use Cyc to guarantee a "natural-like" Interaction/Communication with Human? It is not enough because it is well known in computer science and in AI that the "Organization of Information/Knowledge" is as central and fundamental as the information/knowledge itself [15-19, 23]. If Human (cognition) organizes his $\mathrm{CK}$ in a way that is totally different from the organization used by Cyc (or by other similar axiomatic commonsense-based Ontology) then, the access, the retrieval, the use and the update of the knowledge base will not be the same, and the Interaction/Communication will not be "natural" (human will be frustrated by the behaviour of the computer). Communication/Interaction between humans is possible and efficient because humans share similar CK AND similar 'Knowledge Organization”. "Knowledge Organization" is an inescapable and a fundamental phenomenon. From Cognitive Science and AI, we retain the basic conclusion that Conceptual Structures (like Definition, Canon and Schematic Clusters) are good candidates for CK Organization at the level of concept types.

\section{A solution to the conflict between Formal Ontology and $C K$ in the context of $\mathrm{CHI} / \mathrm{C}$}

In definitive, there isn't a sharp distinction between "Formal Ontology" and "CK Base”: unlike the belief of some philosophers (like B. Smith [20-22]), an Ontology is Knowledge "after all". It is a formalized CK, with a focus on Necessity-Truth and a discard of schematic/situational/typical knowledge. It is argued in previous sections that Knowledge is more important than Necessity-Truth in the context of "full/natural" CHI/C. Therefore, in this context, Formal Ontology can be considered as a special case of CK Base (CK where only definitional/axiomatic knowledge is considered). For $\mathrm{CHI} / \mathrm{C}$ domains that require more $\mathrm{CK}$, i.e. that require schematic/situational/typical knowledge, the domain ontology should correspond to a "full” CK base.

Amine's Ontology Layer allows the creation and use of ontologies that belong to this Ontology-CK base continuum.

\section{Amine's Ontology Meta-Model}

Amine does not provide a specific ontology, but it provides "epistemological constructs" that determine the organization of "multi-lingua conceptual ontology". It 
is the responsibility of the user/designer to use "adequately" these constructs to model her/his ontology according to her/his domain semantics.

A "multi-lingua conceptual ontology", or "CK-based ontology" is a generic hierarchical knowledge base that "defines” the conceptual vocabulary (concept types, relations and individuals) of a domain. Several lexicons (for different languages) can be associated to a CK-based ontology.

The "definition" of a concept type is considered in Amine in a large sense: it corresponds not only to the (classical) type definition, but to the whole CK associated to the type. The CK for each type is organized in terms of different Conceptual Structures (CS). More specifically, Amine's ontology meta-model proposes three kinds of CS for three kinds of CK (CK that is related to each type):

a) Definition: this CS enables the expression of definitional knowledge.

b) Canon: this CS enables the expression of constraint and axiomatic knowledge. It is currently limited to the expression of canonical constraints [25].

c) Schematic cluster (schemata): schematic cluster is an open-end extendible and dynamic set of schemata. Concept, Relation and Schema are the three basic, fundamental and essential constituents of Knowledge. In most cases, we can't reduce the meaning of a concept to a closed and situation-free definition (assuming that such a definition is possible). A concept is like a "coin with two sides": a possible closed-fixed-static side (the definition) and an open-extendibledynamic side (the schematic cluster). Schematic cluster constitutes a fundamental part in the specification of concept meaning. Schema is strongly related to other fundamental cognitive notions like "Patterns", "Conceptual Network”, "Mental Models”, "Situations”, “Contexts” and "Experiences” (chunk of Knowledge) and to various implementations of schema in AI (frames, scripts, situation, schema, MOP, TOP, TAU, etc.). Schemata are high level discrete units of Knowledge (concepts represent the low level discrete units). Schemata are dynamic, interconnected, continually combined in higher schemata that are themselves combined, etc., forming a continuous, dynamic and extendible web of schemata.

At the structural level: an ontology in Amine is a generalization graph where nodes represent Conceptual Structures (CSs). Currently, there are four types of nodes (the first author is working on a fifth type of node: metaphor. See Amine Web Site for more detail). The fourth type of nodes; context node, is not presented in this paper:

a. Type and RelationType nodes: nodes that represent concept type and relation type respectively. These nodes contain the definition of the type (if provided) and/or the canon of the type (if provided).

b. Individual node: a node that represents an individual (an instance) of a concept type. This node contains the description of the individual (if provided).

c. Schema/Situation node: a node that contains the description of a schema/situation.

There are three types of links used in the composition of a "CK-based ontology":

a. Specialization link (s): A type (node) can be specialized by other types (related to them by the (s)pecialization/subtype link). Also, a schema/situation can be specialized by other schemas/situations. 
b. Instantiation link (i): the (i)ndividual link relates an individual (an instance) to its type.

c. Utilisation link $(u)$ : In general, a schema/situation is not indexed under all types contained in it, but only to some of them (determined by the user or by a process that interact with the ontology). The schema is related to these types with (u)se links.

None of the above Conceptual Structures are mandatory: a "minimal" definition of a type is the specification of its super-types (the equivalent of primitive concepts in KLONE family). Then, if a definition is available, it can be specified. Also, schemata/situations can be added to the ontology as more CK is acquired and required. Also, the content of a canon can be augmented by more constraints and axiomatic rules as needed.

By constraining what kind of CK to consider, Amine's user can define and use different kinds of ontologies. For instance, the simple kind of ontologies is "taxonomic ontology"; ontology composed of primitive-types hierarchy. Another kind of Ontology is "terminological ontology" where the hierarchy is enriched by type definitions (and optionally axioms). Lastly, user can define and use "Commonsense Knowledge-based Ontology", where "full" CK is considered; with the use of schematic clusters.

Amine Ontology Layer can be used to develop all these kinds of ontologies.

Ontology modelling language: Amine ontology Layer is not committed to a specific modelling language; any language (CG, KIF, XML, RDF, Frame-Like, etc.) that is interfaced with Java may be used as a modelling language for the description of CS. Indeed, at the implementation level, a description of a CS is declared as "Object” (the root class in Java). However, since we adopt Conceptual Graph (CG) as the basic modelling language in the other layers of Amine (algebraic, programming and multiagents layers), we use also CG as a modelling language in our ontology examples (see next sections and Amine Web Site for more information about this point).

To be able to create/edit/update ontology in Amine, the developer may either (i) use ontology layer's APIs, from a Java program, or (ii) use directly Amine's ontology GUI. Amine Web Site provides examples of ontology creation and update using related APIs from Java programs. The next section presents Amine's ontology GUI/Editor.

\section{Amine's ontology GUI}

Amine's ontology GUI is a multi-view editor (a tree view, a drawing view and a browser view) that allows the creation, consultation, browsing, edition and update of ontology. This section briefly presents these three views. Figure 1 is a snapshot of an ontology edited using the tree view editor. The ontology is visualized in the main frame as a hierarchy. Tree nodes represent CSs nodes of the ontology (each type of node is represented with a different colour). As noted before, a type node can be specialized by other types (and related to them by the (s)pecialization link). For 
instance, PetrolEngine is a specialization of Engine and Gasoline. Also, a type can be related to several individuals (by (i)ndividual link) and it can be related to several schemata/situations (by (u)se link). For instance, situation SIT\#7 is associated to the type Container. Also, a situation can be specialized by other situations. For instance, SIT\#7 is specialized by SIT\#9.

The user can select a specific language (from the languages list associated to the ontology) to get the ontology according to the selected language. If required by the user, the content of a node is visualized in an auxiliary frame. Some auxiliary frames are shown in Figure 1: one frame shows the content of the type node "PetrolEngine" and two other frames show contents of two situations. A Type node contains type's definition (if provided) and canon (if provided). For instance, "PetrolEngine" has only a definition. The description of a CS (see the three auxiliaries' frames in Figure 1) is a CG that is displayed in multi-lingua multi-notations CG editor (Figure 1): CG can be visualized according to the selected language (one tab for every language) and according to the selected CG notation (one tab for every notation: Linear form, CGIF and Graphical form).

Ontology's GUI provides also the possibility to display the super types of a given type. For example, the frame "Super Types" shows the super types of the type "Steel". It is also possible to edit synonyms in all available languages for a given type. For instance, the Frame "Edit Synonyms for the type: Container" shows synonyms of the type "Container" in both English and French.

The second possibility is to edit/consult ontology in "Draw View" Editor. Using graphical components (graphical nodes for CSs and arrows for links), this view allows to use the same functions as those available in tree view mode. Of course, the classical graphical operations are available (edition, selection, move, cut/copy/past, etc.) and any change (addition, suppression, modification of nodes and/or links) made on either modes affect directly the ontology and thus is visible in both modes. Also, user can zoom in/out the ontology, she/he can fix the vertical spacing between ontology nodes and she/he can locate the position of a specific type or an individual node in the ontology.

To produce an automatic drawing view of an ontology from its tree view, we use the basic Sugiyama algorithm ${ }^{2}$ that enables automatic drawing of a hierarchical graph. An improvement of automatic drawing of ontology is underway.

For a large ontology, the user may have trouble reading the ontology from the drawing view. To accommodate this problem, a "Browser View Editor" is provided that allows the user to focus on a particular area of the ontology: a) the Browser Editor allows getting the neighbourhood of a particular node in the ontology, b) then, functions "expand" and "collapse" allow user to explore neighbourhood of any node in the Browser view. With expand/collapse functions, user can perform selective browsing of her/his ontology.

${ }^{2}$ plg.uwaterloo.ca/ itbowman/CS746G/Notes/Sugiyama1981 MVU/ 


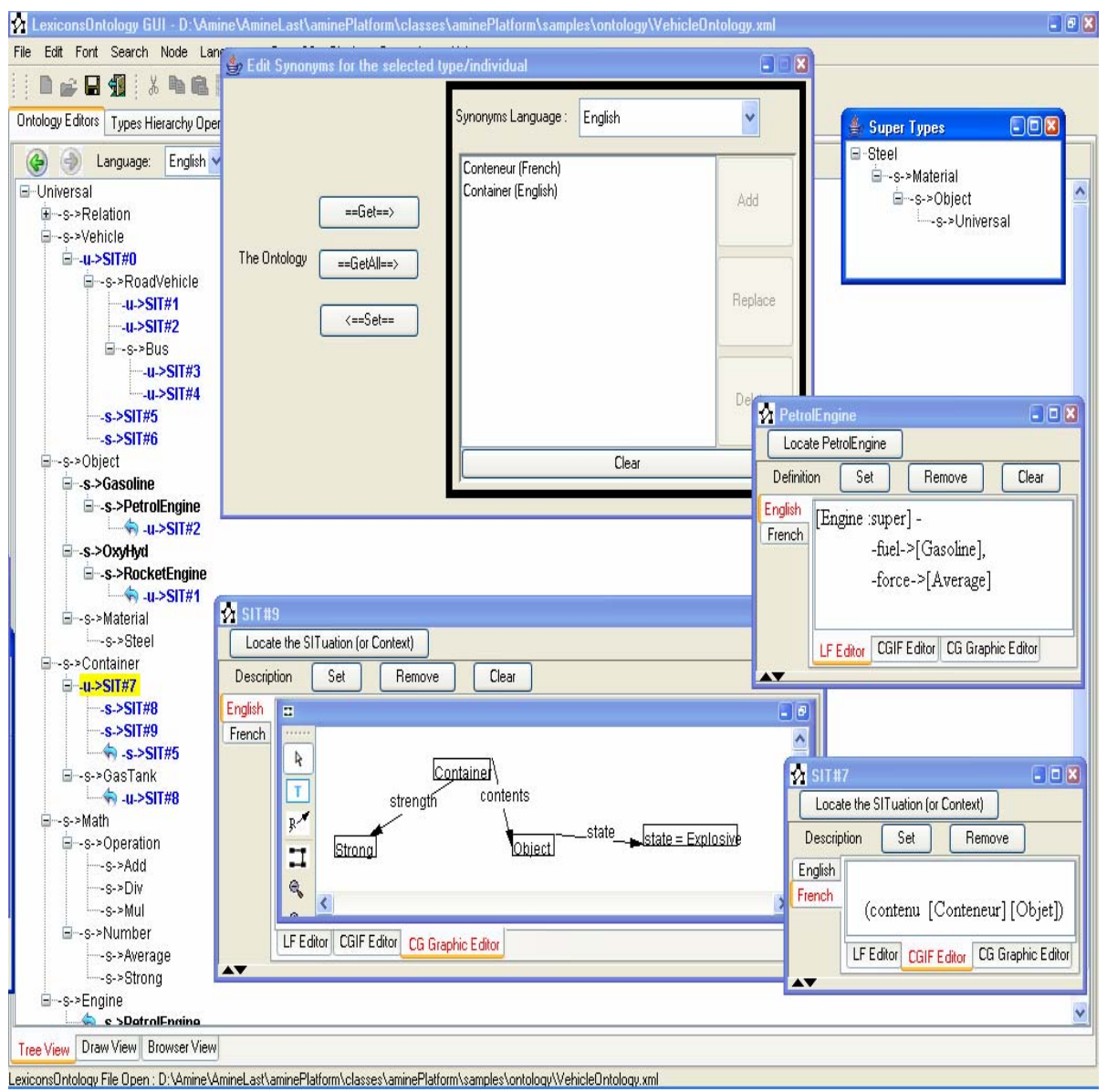

Figure 1: Ontology Tree View Editor

\section{Ontology related processes}

This section presents a short description of Amine's ontology related processes (see Amine Web Site for a detailed description). Unlike most of the "ontology dedicated tools/systems”, Ontology processes in Amine are not limited to Classification (and related processes). Indeed, since Amine considers Ontology as a special case of CK Base, several CK Base processes are also offered (Classification, Information Retrieval, Dynamic Knowledge Integration, Elicitation and Elaboration). Here is a brief description of these processes:

- Classification process uses subsumption operation to classify a description in an ontology.

- Information retrieval process (IR) uses the classification process and searches to know if the specified description is contained or not in the ontology/memory. The aim of IR is not to answer by "yes" or "no", but rather to situate the specified 
description in the ontology; to determine its neighbourhood: which nodes are "fathers" (minimal generalizations of the specified description), which nodes are "children" (minimal specialization of the specified description) and which node is equal to the specified description. Here is an example that illustrates the use of IR from Prolog+CG program/console (see the companion paper for more detail on Prolog+CG [14]):

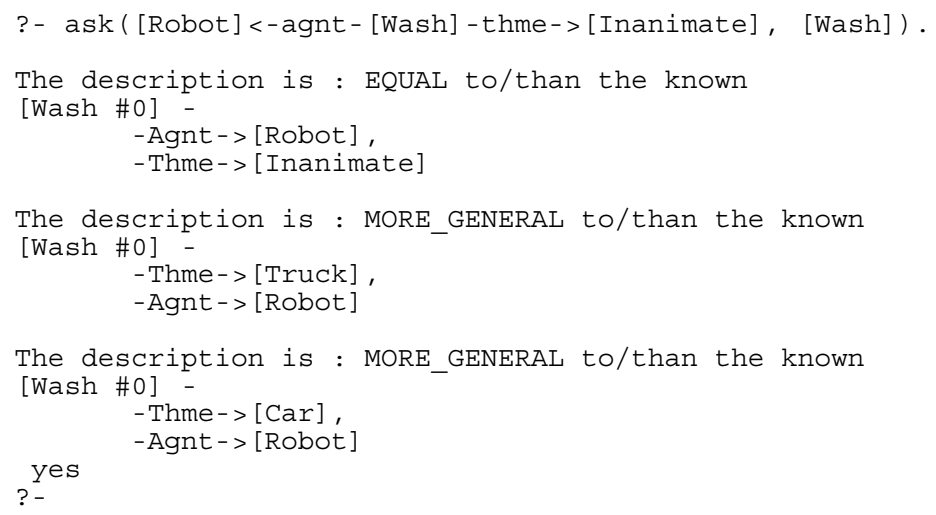

- Dynamic integration process concerns especially the integration of a description of type definition or of schema/situation. Dynamic integration process (or knowledge acquisition) performs automatic and incremental integration of new information in the ontology. The use of this integration process involves a similarity and generalization based construction and re-organization of the ontology. Contrary to classification, dynamic integration process is a "constructive learning task": it may create a new concept as a result of comparison between other concepts. Dynamic integration process is related to concept learning and machine learning in general, and to dynamic memory models in particular [15, 19].

- Elicitation process is an interactive process that helps a user to make his description $\mathrm{D}$ more precise and more explicit. For instance, assume that $\mathrm{D}$ is: [Vehicle]-engn->[Engine]. The user is asked if, by using Engine, he intends PetrolEngine or RocketEngine. The user may ask for the definition of the specified subtype (to decide if the proposed subtype is indeed the intended type). If the user selects one subtype (PetrolEngine for instance), the description is updated (Engine will be replaced by PetrolEngine): [Bus]-engn-> [PetrolEngine]. The process will continue iteratively: it considers all the types used in the current description including the new types (PetrolEngine could be replaced by a more specific type). Beside this type-directed elicitation process, Amine provides a situation-directed elicitation process: while type-directed elicitation operates at the concept level (change of concept types), situation-directed elicitation operates at the structural level: the current description is integrated in the ontology, using the classification process, in order to situate the description in the ontology and to identify its neighbourhood; to determine situations that are more specific to the current description. The user is then asked if one of these situations fits his/her 
intended description. For instance, situation-directed elicitation may start from the result of the above type-directed elicitation ([Bus]-engn->[PetrolEngine]). The user gets then a request from Amine:

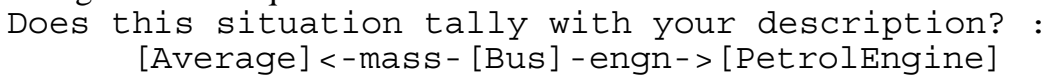

If the user's response is yes, the current description is replaced by the selected situation and the process continues with situations that are more specific than the selected situation. In this sense, the user is involved in an elicitation process.

- Elaboration process is introduced below.

See Amine Web Site for more detail about these processes. Classification, Information Retrieval and Dynamic Knowledge Integration will be described in more detail in a forthcoming paper.

Elaboration process uses the inheritance mechanism to provide more information about a description D. We differentiate between "deductive elaboration" which uses inheritance of type definitions and "inductive/plausible elaboration" which uses inheritance of schemata/situations ("plausible elaboration" because a situation provides only typical/plausible information about a type, contrary to a definition). A mixture of the two kinds of elaboration is possible.

Elaboration process is an interactive process: user provides a description D (of a proposition or of a situation). Then she/he asks the system to "elaborate/explicitate" D; "Can you provide more information about D?" The system “elaborates" by applying the inheritance mechanism to join "relevant information", from the current ontology, to D. For instance, if a concept type used in D has a definition, then this definition is joined to D. After that, user can ask the system to elaborate D further involving other joins (specialization), etc. Elaboration process is useful for users that are not familiar with (or expert in) a domain (and its conceptual vocabulary).

Let us consider the following simple example (Figure 2): the process starts with the user's description D (for instance, $\mathrm{D}=$ [Bus]-engn->[PetrolEngine]). The system locates, in the ontology, the definition of PetrolEngine and joins it to D (Figure 2.a). If the system is asked by the user to elaborate further, the process continues with the join of the Gasoline definition (Figure 2.b). The elaboration process will terminate after that because all superTypes of types present in the (new) formulation of D have no definition.

A similar treatment is performed by "plausible elaboration" which uses inheritance of schemata/situations. For instance, Figure 2.c shows the result of the specialization of the current description (Figure 2.b) by the join of an inherited schema/situation (schema that is associated to a superType of a type specified in the description).

Note that an inherited situation that can't be joined with the current description is ignored.

(a)

(b)

\begin{tabular}{|c|c|}
\hline $\begin{array}{c}\text { [Bus]-engn- }>\text { [PetrolEngine]- } \\
- \text { fuel- }>\text { [Gasoline], } \\
- \text { force- }>\text { [Average] }\end{array}$ & $\begin{array}{c}\text { [Bus]-engn- }>\text { [PetrolEngine]- } \\
\text { fuel- }>\text { [Gasoline]-state- }>\text { [State }=\text { Volatile }], \\
\end{array}$ \\
& - force- $>$ [Average $]$ \\
\hline
\end{tabular}




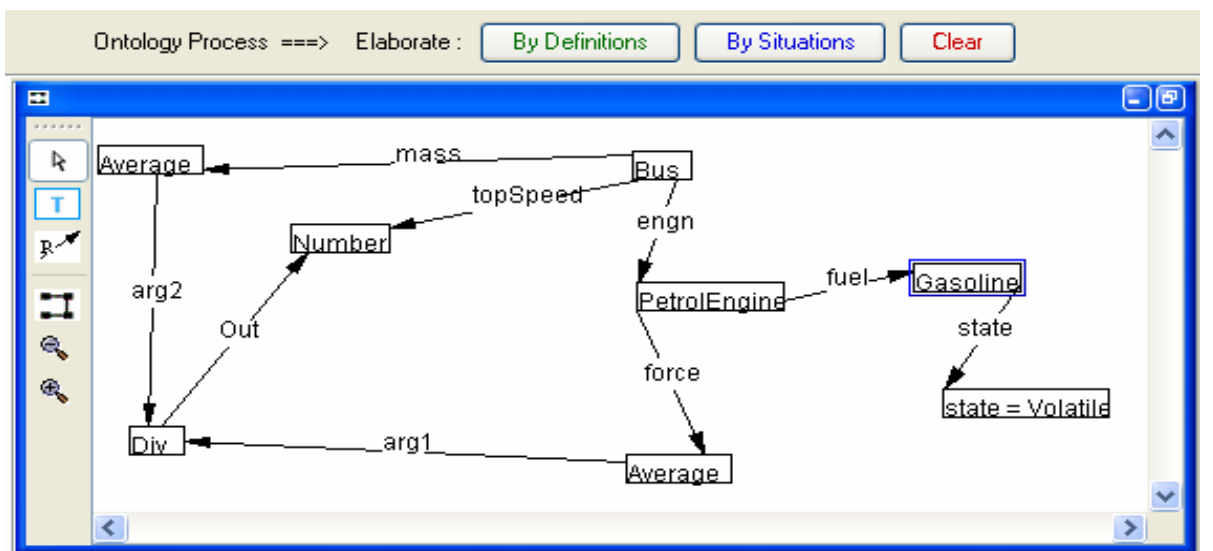

Figure 2: elaboration process

\section{Conclusion, current and future work}

Formal Ontology is a formalized CK, with a focus on Necessity-Truth and a discard of schematic/situational/typical knowledge (which corresponds to a broader view and approach to CK). It is argued in this paper that Knowledge is more important than Necessity-Truth in the context of "full/natural" CHI/C. Therefore, in this context, Formal Ontology can be considered as a special case of CK Base (CK where only definitional/axiomatic knowledge is considered). For $\mathrm{CHI} / \mathrm{C}$ domains that require more CK, i.e. that require schematic/situational/typical knowledge, the domain ontology should correspond to a "full" CK base.

Amine's Ontology Layer allows the creation and use of ontologies that belong to this Ontology-CK base continuum.

Amine's ontology layer presents a specific ontology meta-model and various related processes (edition, elaboration, elicitation, classification, information retrieval and dynamic integration). All these processes are considered as "basic" ontology processes; they can be used or extended in many ways according to the need of the application's domain.

Amine platform, with its Ontology Layer, constitutes a starting point for "integrative development" of ontology-based intelligent systems and intelligent agents. Many works are still to be done however, in several directions ("Applied Ontology", Commonsense $\mathrm{KB}$, improving ontology editors and processes, investigating the links between ontology and metaphoric knowledge, between ontology and dynamic memory, development of intelligent agent with ontology/memory, etc.).

\section{References}

[1] Baader, F., et al. (eds.): Description Logic Handbook, Cambridge University Press, 2002 
[2] Brachman R. J. and J. G. Schmolze, An Overview of the KL-ONE Knowledge Representation System, Cognitive Science 9, 171-216, 1985

[3] Brewter C. and K. O’Hara, Knowledge Representation with Ontologies: The Present and Future, p. 72-81, IEEE Intelligent Systems, January/February 2004

[4] Dölling J., Commonsense Ontology and Semantics of Natural Language, 1993 //www.uni-leipzig.de/ doelling/publikationen.html

[5] Doyle J. and R. S. Patil, Two theses of knowledge representation, Artificial Intelligence 48, pp. 261-297, 1991

[6] Eysenck M. W., A Handbook of Cognitive Psychology, Lawrence Erlbaum Associates, 1984

[7] Farrar S. and J. Bateman, General Ontology Baseline, 2004, //www.sfbtr8.uni-bremen.de/project.html

[8] Fauconnier G. and M. Turner, The Way We Think, Basic Books, 2002

[9] Fensel D. and al., OIL: An Ontology Infrastructure for the Semantic Web, IEEE Intelligent Systems, p. 38-45, March/April 2001

[10] Guarino N. Formal Ontology and Information Systems. 1998 www.ladseb.pd.cnr.it/infor/Ontology/ontology.html

[11] Guarino N., Formal Ontology, Conceptual Analysis and Knowledge Representation. 1995 see URL [10]

[12] Guarino N. The Ontological Level, 1993. see URL [10]

[14] Kabbaj A., Development of Intelligent Systems and Multi-Agents Systems with Amine Platform, 2006 (in this volume).

[15] Kolodner J. L. and C. K. Riesbeck (eds.), Experience, Memory, and Reasoning, Lawrence Erlbaum Associates, 1986.

[16] Lakoff G., Women, Fire, and Dangerous Things: what categories reveal about the Mind, University of Chicago Press, 1987.

[17] Luger G. F. and al., Cognitive Science, Academic Press, 1995

[18] Murphy, G. L., The big book of concepts, MIT Press, 2002

[19] Schank R. C., Tell Me a Story: A new look at real and artificial memory, MacMillan, 1991.

[20] Smith B., Formal Ontology, Common Sense and Cognitive Science, 1995 http://ontology.buffalo.edu/smith/articles/

[21] Smith B., Ontology, 2003. See URL in [22]

[22] Smith B., Beyond Concepts: Ontology as Reality Representation, 2004. See URL in [22]

[23] Sternberg R. J. (Ed.), Thinking and Problem Solving, Academic Press, 1994

[24] Sowa J. F., Knowledge Representations, Brooks Cole Publishing, 2000. See also www.jfsowa.com

[25] Sowa J. F., Conceptual Structures: Information Processing in Man and Machine, Addison-Wesley, 1984 